\title{
Aqueous Nanoscale Systems
}

\author{
Sylvie Roke*
}

\begin{abstract}
In the past five years the Laboratory for fundamental BioPhotonics (LBP) has worked on developing new technology that can access the molecular structure and nanoscale properties of buried aqueous interfaces and aqueous solutions. Using these methods a better understanding of the important role that water plays in (nanoscale and interfacial) processes can be obtained. These processes include the long-range interaction of ions with water, structural and charge anomalies of the hydrophobic/aqueous interface, the formation and stabilization of amphiphilic aqueous droplet interfaces, the formation and molecular properties of the electric double layer, as well as membrane structure and hydration. The result of our work on these themes is summarized for this special issue article.
\end{abstract}

Keywords: Droplets · Interfaces · Nonlinear imaging · Nonlinear light scattering $\cdot$ Membranes · Water

\section{Introduction}

Water. No substance on earth is so intimately linked to our well-being. Without it, we die. On a more scientific level, without water, membranes - the structures that provide the architecture of our cells and organelles - cannot function. Charges and charged groups cannot be dissolved, selfassembly cannot occur, and proteins cannot fold. Apart from the intimate link with life, water also shapes the earth and our climate. Our landscape is formed by slow eroding/dissolving processes of rocks in river and sea water; aerosols and rain drops provide a means of transport of water. Our society depends on products that all relate to water and aqueous systems, such as food products, medicine, and consumergoods.

In most of the above mentioned systems it is the interfacial region (of the membrane, the droplet, or the particle) that determines much of the physical, chemical, biological, and geological properties. ${ }^{[1,2]}$ Interfacial water is often considered in one of two ways: As a background, describable by a single parameter, or simply omitted. ${ }^{[3,4]} \mathrm{Al}$ ternatively, it is studied in great detail in an

\footnotetext{
${ }^{\star}$ Correspondence: Prof. Dr. S. Roke

Laboratory for fundamental BioPhotonics (LBP)

Institute of Bioengineering (IBI)

and Institute of Materials Science (IMX)

School of Engineering (STI)

and Lausanne Centre for Ultrafast Science (LACUS)

École Polytechnique Fédérale de Lausanne (EPFL)

$\mathrm{CH}-1015$ Lausanne

E-mail: sylvie.roke@epfl.ch
}

environment or condition that is precisely defined but so oversimplified that it has not much to do with the real world. Aqueous interfaces are mostly studied in vacuo, or as a planar water/air interface. ${ }^{[5,6]}$

However, interfacial water occurs on different length scales, from sub-nanometer to micron sized (corrugations, organelles, membranes, liposomes), and is often buried inside another solid or liquid environment that is not at all comparable to vacuum or air. This absence of molecular knowledge of realistic interfaces is due to a lack of tools that can access buried nanoor microscopic interfaces in liquids and solids.

In the past years, we have obtained a better understanding of the important role that water plays in interfacial processes, such as the long-range interaction of ions with water, structural and charge anomalies of the hydrophobic/aqueous interface, the formation and stabilization of amphiphilic aqueous interfaces, the formation and molecular properties of the electric double layer, as well as membrane structure and hydration. We have achieved these new insights by developing methods that can access molecular and nanoscale properties of aqueous systems and interfaces more accurately, as well as probing multiple time and length scales simultaneously. In what follows we will first outline our strategy, and then consider the above mentioned processes in more detail. Finally, we highlight several differences between nanoscopic and macroscopic aqueous interfaces.

\section{Probes for Nanoscopic Aqueous Systems}

Probing aqueous systems on different length scales requires methods that cover different length scales, such as linear and nonlinear light scattering measurements: dynamic light scattering $(\sim \mathrm{mm}, \mathrm{s})$, femtosecond (fs) second harmonic scattering (sensitive to nanoscale information from the scattering pattern), vibrational sum frequency scattering (spectral information, with sub-ps dynamical information and nanoscale information from the scattering pattern), and multiphoton imaging (with a $\sim 200 \mathrm{~nm}$ resolution and $500 \mu \mathrm{m}$ field of view; and $\mu$ s acquisition times). A large part of our research effort has been aimed at developing those methods. We have also developed the necessary nonlinear optical models and theories that allow us to access detailed molecular level information about interfacial processes. Fig. 1 shows an illustration of the experimental methods with energy level schemes for second harmonic (SH) and sum frequency (SF) generation. In both methods the production of a coherent $\mathrm{SH}$ or SF photon only occurs when non-centrosymmetric molecules are spatially distributed in a non-centrosymmetric way. This allows one to selectively probe various specific structures, such as polar fibrils (e.g. microtubules or collagen), aqueous interfaces, electric field induced orientation of water molecules or the conformation of moleculargroups.

In conjunction with the optical methods we use a nanoparticle/droplet platform that allows probing solid and liquid interfaces around micron or nanoscale particles/ droplets in solution. Nanodroplets have a surface to volume ratio that is $\sim 4$ orders of magnitude larger than that of a planar macroscopic interface. Thus, by using nano-interfaces we can dramatically increase the efficiency and accuracy of an interface measurement. Furthermore, preparation procedures can be done entirely in the bulk phase, and require a small sample volume of typically 50-100 microliter. This allows for a dramatic reduction of impurity issues and unwanted oxidation induced by ambient air. It also reduces restrictions for 


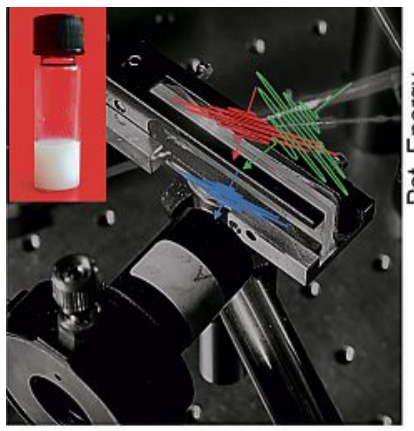

a

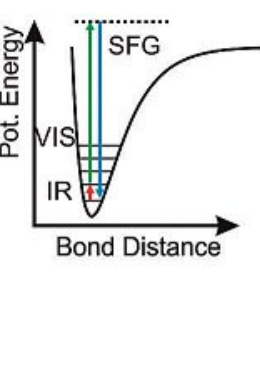

.

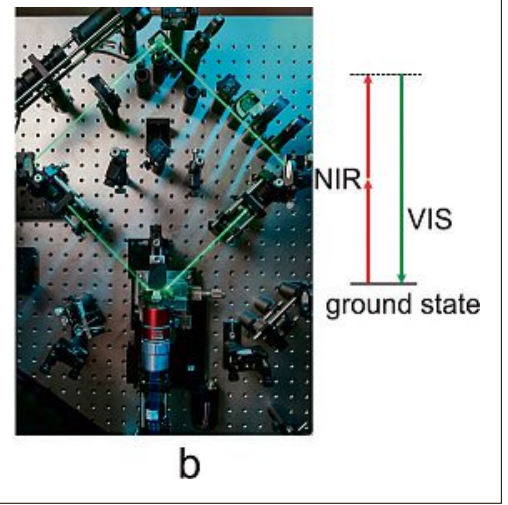

Fig. 1. Illustration of methods. (a). Sum frequency scattering. Sketch of the beam geometry and energy level scheme. A combined IR and Raman transition occurs, which is only allowed in a non-centrosymmetric environment. (b) Photo (Alain Herzog, EPFL) of the second harmonic microscope and energy level scheme for non-resonant second harmonic generation. This process is elastic and has the same symmetry selection rules as sum frequency generation.

optical probes as the scattering experiments can be performed with the incoming beams in transmission geometry as opposed to more complex reflection schemes. In addition, although many of the nanoscopic systems studied can be considered as model systems, they also occur in living systems with important biological functions (think of lipid droplets and liposomes).

In what follows, some exiting advancements that were achieved in the Roke lab at EPFL are outlined, first regarding experimental and theoretical method development, and then regarding the advancements in understanding the molecular architecture of aqueous systems.

\section{Method Development}

We have laid a technological foundation by developing time- and frequency resolved femtosecond (fs) vibrational sum frequency scattering (SFS), a combination of light scattering and nonlinear spectroscopy in aqueous solutions (see refs [7-11], as illustrated in Fig. 1a). Thanks to the symmetry selection rules for second-order nonlinear optical processes, SFS allows to measure the molecular surface structure, morphology and chirality of nano- and microscopic objects in solution. A variety of systems, such as polymer particles in a solid matrix, ${ }^{[9]}$ particles in solution, ${ }^{[12]}$ oil droplets in water, ${ }^{[7]}$ water droplets, ${ }^{[13]}$ a micro-jet ${ }^{[11]}$ and liposomes ${ }^{[14]}$ in aqueous solution were characterized, often with surprising outcomes. Nanoscale curved interfaces do not always behave in the same way as extended planar interfaces.

More recently, we have developed a new instrumental approach for fs-second harmonic scattering (SHS, a non-resonant form of SFS, employing two identical frequencies, and addressing the electronic states of a molecule rather than the vibra- tional levels, Fig. 1b). This has led to instrumentation with an increased throughput by three orders of magnitude. [15] The enhancement enables the probing of the orientational ordering of water at nanoscopic aqueous interfaces on millisecond time scales.

The technological developments go hand in hand with theoretical work: We have published a series of papers laying down fundamental scattering theorems ${ }^{[16-20]}$ that can be combined with molecular dynamics simulations. ${ }^{[21,22]}$ Our models are available as freeware.

One recent example that stands out is the measurement of unique values of the surface potential from angle resolved polarimetric SHS. ${ }^{[23]}$ The method is illustrated in Fig. 2. Fig. 2 shows two SH scattering patterns of $100 \mathrm{~nm}$ diameter liposomes in dilute aqueous solution. The two independent scattering patterns are recorded with the optical fields polarized in different directions. The patterns can be described by an optical model that uses the surface potential as one of two fit parameters. The obtained surface potential values are plotted in the right panel and compared to

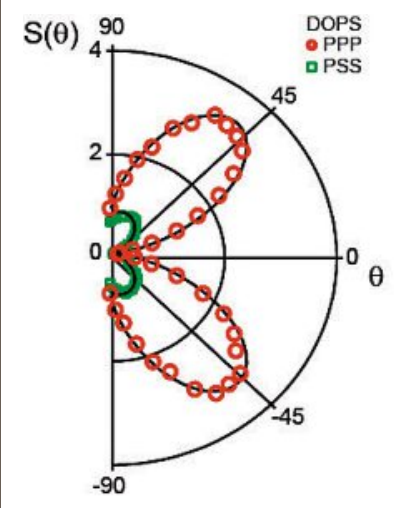

values from different surface models. In contrast to other methods, no mean-field assumptions about the interfacial structure are needed. The portable table top method can be applied to any type of particle in any liquid or solid medium.

In addition, the principles of nonlinear light scattering can be transferred to microscopy, allowing for high-throughput wide field multiphoton microscopy. ${ }^{[24-26]}$ Our approach relies on reducing the repetition rate of the femtosecond laser pulse train (from the $\mathrm{GHz}$ range), while optimizing the pulse energy, so that the throughput can be optimized. As the number of emitted $\mathrm{SH}$ photons scales quadratically with the pulse energy and linearly with the repetition rate, and the lower repetition rate ensures a reduced heat load of the aqueous system, a higher signal to noise level is achieved, [24] and photodamage effects are reduced significantly in living systems (allowing us to increase the dwell time by a factor of $\sim 10^{6[26]}$ ). Fig. 3 shows the improvement in throughput compared to scanning confocal second harmonic imaging. Single living mammalian neurons can now be imaged with second harmonic generation on the sub-cellular level with sub-second acquisition times, ${ }^{[24]}$ and the translational and rotational diffusing of particles can be measured inside living cells with single shot (5 microseconds) accuracy. [25]

\section{Aqueous Systems}

To advance our molecular level understanding of complex aqueous systems we have studied several phenomena that capture essential elements of biochemical processes. Organized in order of increasing complexity these are: aqueous electrolyte solutions, hydrophobic aqueous interfaces, charged amphiphilic interfaces, electric double layer and hydrophobicity, and membrane interfaces.

Aqueous electrolyte solutions form the matrix of life. Ions interact with water in

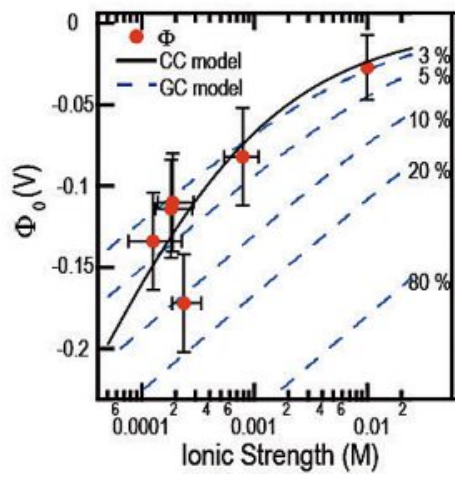

Fig. 2. Surface potential measurements. (a). SHS patterns of liposomes in two different polarization combinations. Using the framework of refs $[19,23]$, the two scattering patterns can be described theoretically using the surface potential and the surface susceptibility as sole unknowns. The procedure allows for the extraction of unique surface potential values. 


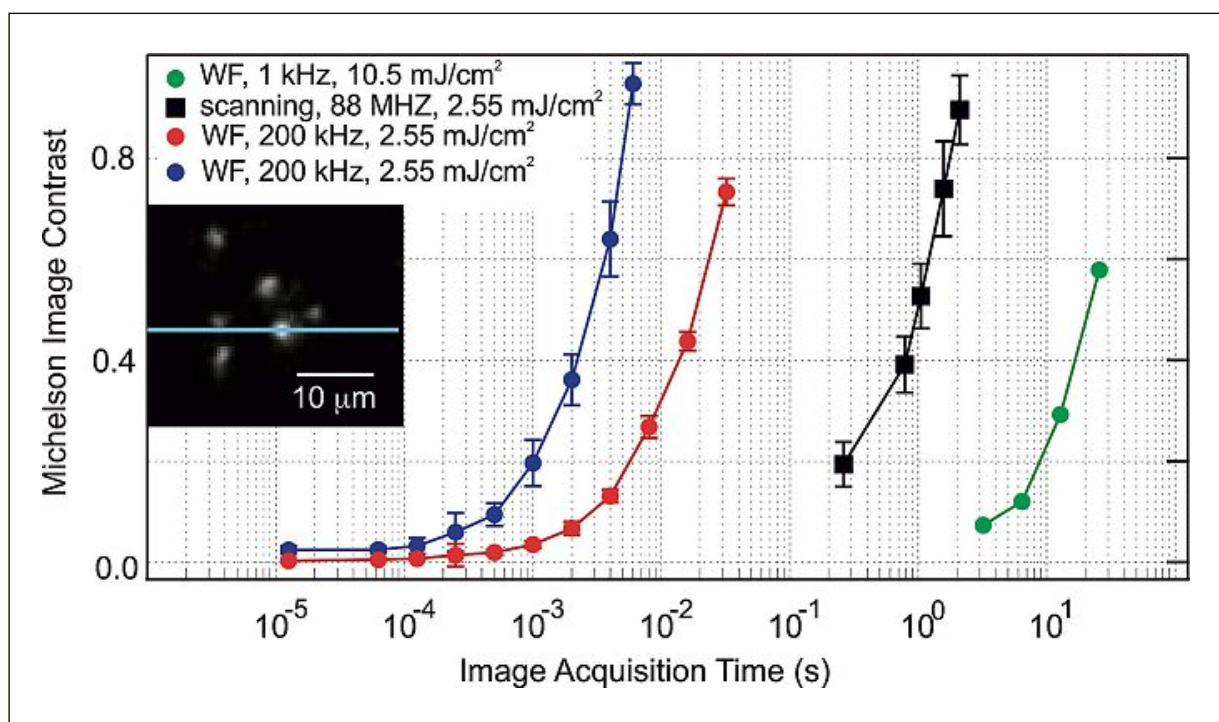

Fig. 3. Measured second harmonic imaging throughput. Measured Michelson contrast in images recorded from the same position of the same $100 \mathrm{~nm} \mathrm{BaTiO}{ }_{3}$ particle sample in four different systems: wide-field $(200 \mathrm{kHz}$, gated detection as proposed here, blue and red curves, using different camera settings), a scanning microscope (Leica TCS SP5 with $1028 \mathrm{~nm}, 88 \mathrm{MHz}, 190$ fs laser pulses illumination, a 1.2 NA 20x water immersion objective, a scanning rate of $1000 \mathrm{~Hz} /$ line, image size of $256 \times 256$ pixels, and collecting NA of 0.9 ), and a wide-field $1 \mathrm{kHz}$ geometry with a normal CCD camera. The used pulse power and repetition rate are given in the legend. The inset shows an image of the nanoparticle sample corresponding to the red data point with the largest contrast.

many ways, changing dipole orientation, inducing charge transfer, and distorting the hydrogen-bonding network. These effects have been studied in experiments probing e.g. vibrational dynamics, dielectric responses, infrared and Raman signatures and computer simulations. All these studies have shown that ion-water interactions are short-range affecting the structure of water in the first, second and at most the third hydration shell. However, such experiments and simulations are biased towards detecting short-range perturbations. Using the unique sensitivity of the fs-SHS instrumentation and the property of nonlinear light scattering experiments to be uniquely sensitive to tiny perturbations on nanoscop-
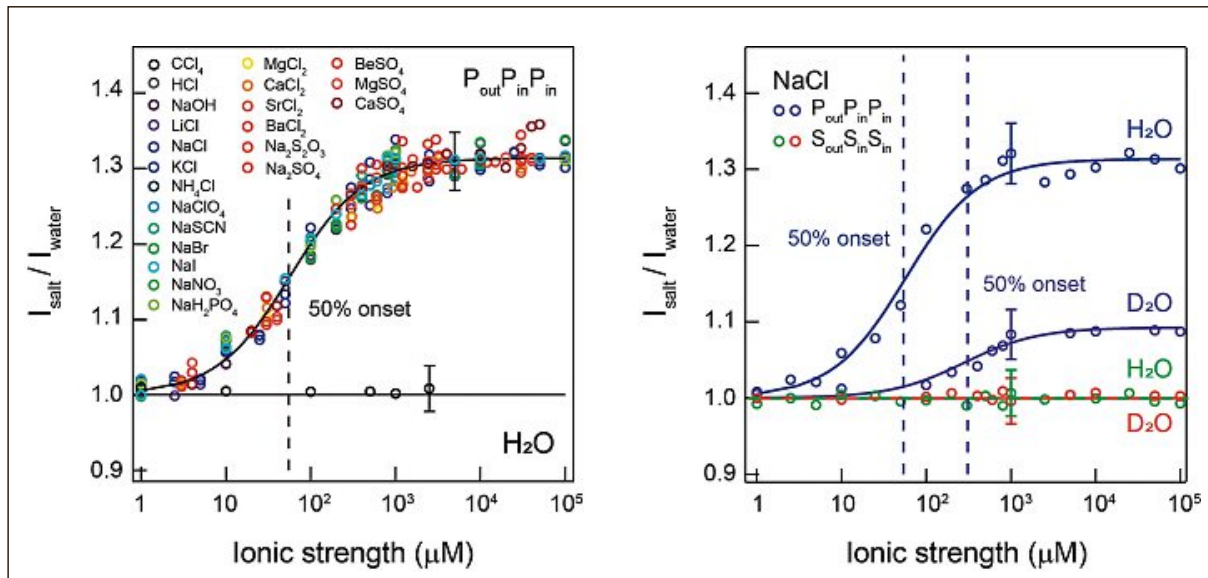

Fig. 4. Electrolytes in water: Left: fs-SHS data of aqueous solutions, showing changes in the orientational order at concentrations $<10 \mu \mathrm{M}$. Right: Large differences are observed between light and heavy water indicating the importance of nuclear quantumeffects. ference between the surface and the bulk of the electrolyte solution. ${ }^{[27]}$ The observed phenomena offer unique insights into the nanoscale properties of liquid water and electrolyte solutions that is explored further in studies that involve both theory ${ }^{[22,28,29]}$ and experiment. ${ }^{[30]}$

In a second set of experiments we addressed the question: "Does water interact differently with positive or negatively charged ions?" This question is motivated by the observation that in biological systems interfaces are generally negatively charged or charge neutral. In addition, hydrophobic interfaces carry an apparent negative charge. This suggests that there is a difference between the hydration and surface structure of cations and anions. Combining Raman hydration shell spectroscopy with SHS and SFS to investigate the molecular interactions with water in solution and at oil nanodroplet/water interfaces, we mapped the interaction of tetraphenyl ions with water. The chosen ions, sketched in Fig. 5a, that are either cationic, with an $\mathrm{As}^{+}$ion as the core of the molecule or anionic, with a $\mathrm{B}^{-}$ion as the core of the molecule have virtually identical sizes, structures and polarizabilities. As such, the electrostatics of the interaction with water and an aqueous interface should be identical. The spectroscopic data, however, shows remarkable differences, as can be seen in Fig. 5b. Comparing molecular structures and the vibrational signatures of the hydrogen bonding in the spectra, we derived that the solvation of anions is slightly more energetically favorable than the solvation of cations. This difference is caused by the different interactions at work. Water-ion interactions can either be dipole-charge interactions, or comprised of hydrogen bonds between the ions and the water molecules. The first interactions have a different orientation, depending on the charge sign of the ion, while the hydrogen bonds are always directed in the same way, with the hydrogen donor of a water molecule directed towards the phenyl ring of the molecular ion. As such, for a negative ion both interactions will be cooperative, while for the cation they will be anticooperative, leading to a slightly less favorable solvation energy of the anion over the cation. At the interface, this results in the cations being more readily solvated by the oil phase.

Hydrophobic aqueous interfaces are key to understanding interactions between water and macromolecular systems and can be prepared by dispersing pure hydrophobic oil nanodroplets in water ${ }^{[31-35]}$ or water droplets in oil.[13] Electrokinetic mobility measurements show that hydrophobic droplets in water are negatively charged and that the charge increases dramatically when the $\mathrm{pH}$ of the solution is 


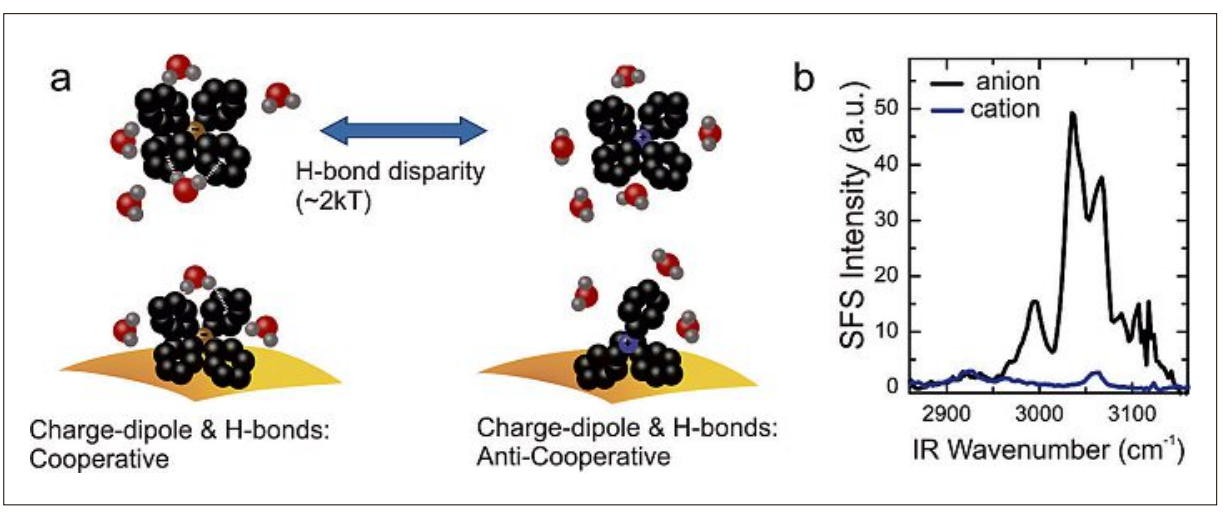

Fig. 5. Charge asymmetric behavior of water. a) Cartoons illustrating the solvation of molecular tetraphenyl ions that have a virtually identical chemical structure and polarizability but a different charge. For the anions hydrogen bonds and dipole-charge interactions can be optimized with water oriented in the same direction. For cations each interaction energy minimum requires a different water orientation. b) SFS spectra of the molecular anion (black) and the molecular cation (red) on an oil nanodroplet in water. The spectral region below $2960 \mathrm{~cm}^{-1}$ represents the vibrational modes of the oil, while the spectral region above $2960 \mathrm{~cm}^{-1}$ displays the structure of the molecular ions, which are seen to be remarkably different.

increased. ${ }^{[31-33]}$ This unusual behavior is commonly explained by the presence of hydroxyl ions at the interface. We observe, however, no $\mathrm{pH}$ dependent accumulation of $\mathrm{OH}^{-}$at the interface ${ }^{[33]}$ and can explicitly exclude surface active impurities as the major driving force for charge accumulation. ${ }^{[32]}$ By analyzing our data with nonlinear light scattering theory in combination with molecular dynamics simulations that include charge transfer effects, we suggest that rather than ionic adsorption, charge transfer between water molecules is responsible for the observed phenomenon. ${ }^{[31]}$

Charged amphiphilic interfaces are aqueous interfaces that contain both hydrophobic and hydrophilic groups. We have prepared droplets in water that contain various amphiphilic molecules, such as neutral and ionic surfactants, as well as phospholipids. By measuring the molecular composition of the droplet oil surface, the surface amphiphiles and the adjacent water as well as the interfacial charge, we obtain information of different length scales that can be combined in a unified interfacial structural picture. With this approach we found that long chain alkane $\left(>\mathrm{C}_{8}\right)$ oil droplets minimize their interfacial free energy with surface oil molecules that lie flat on the surface of the oil droplets. ${ }^{[21,36]}$ Positively and negatively charged ionic amphiphiles interact remarkably different with hydrophobic/ water interfaces. ${ }^{[37]}$ Studies of the interfacial structure of sodium dodecylsulfate and dodecyltrimethylammonium, showed distinct behaviors for negatively charged amphiphiles and positively charged ones. The negatively charged amphiphiles are more hydrophilic than the positively charged ones, as the dipole-charge interaction between the negative amphiphile and the water and the hydrogen bonding of water with the head group are pointing in the same di-

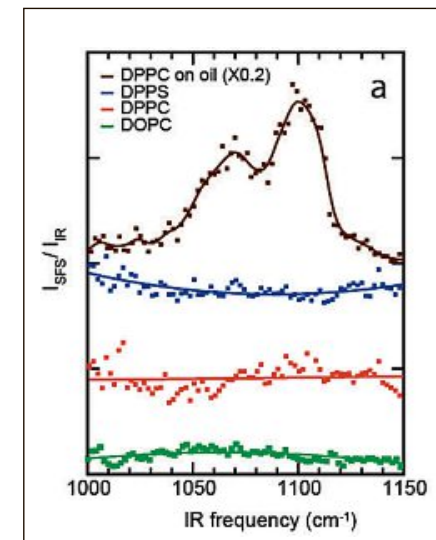

that indicate the opposite are known. ${ }^{[40-42]}$ These effects have not been quantified, however. We have started to do this with our multiscale toolbox and find that the nanometer length scale and the hydrophobicity of the cations is crucial in determining whether the ions only influence the water structure in the interfacial region or also the surface itself. ${ }^{[43]}$ In addition, the ability to determine the average value of the surface potential is a great help. ${ }^{[23,44]}$

Membrane interfaces: Lipid droplets and liposomes. The interfacial environment of membranes is crucial for transport, signaling and the function of organelles. This comprises the molecular structure of the lipids as well as the hydrating water. We have developed a lipid droplet-like system comprised of oil droplets surrounded by a lipid monolayer that both mimics the naturally occurring adiposomes and at the same time presents a tunable, large surface to volume ratio (but small volume) system for molecular studies of membrane systems (Fig. 6). ${ }^{[45,46]}$ These droplets were used to study the membrane structure of liposomes in solution and it was found, surprisingly, that the inner and outer leaflets of liposomes have identical numbers of lipids. The significant difference in area $(\sim 15 \%$, which was always assumed to be filled with lipids) is filled up with hydrating water molecules. ${ }^{[14]}$ Specific head group interactions may cause transmembrane asymmetries to occur. In addition surface potentials of liposomes were determined, and it was concluded that the commonly employed mean field model cannot be used to describe the electrostatic properties of these interfaces. ${ }^{[23]}$ Instead charge condensation plays a very important role. ${ }^{[44]}$

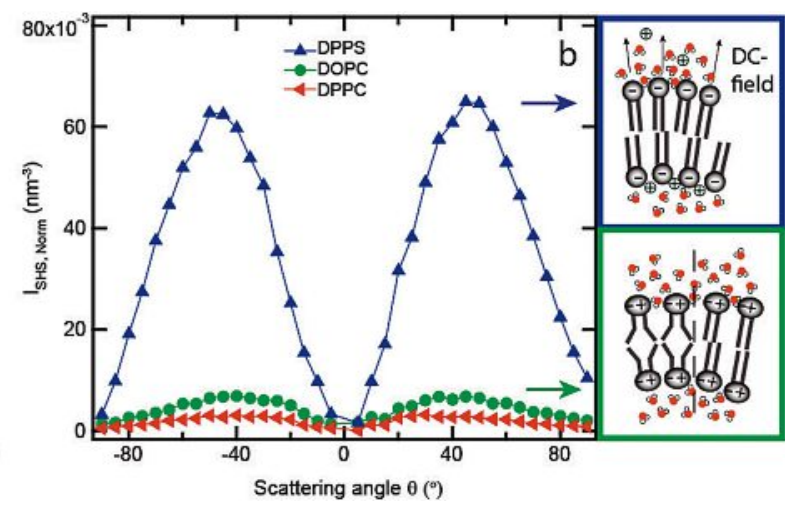

Fig. 6. Liposome transmembrane asymmetry. a) SFS spectra of $\sim 50 \mathrm{~nm}$ radius DPPS (blue), DOPC (green), and DPPC (red) liposomes in $\mathrm{D}_{2} \mathrm{O}$, probed in the $\mathrm{P}-\mathrm{O}$ stretch region together with an SFS spectrum of hexadecane oil droplets covered with a DPPC monolayer (top trace). The SFS data are offset vertically for clarity. It can be seen that, in contrast to the oil droplet covered with lipids, there is no detectable transmembrane asymmetry of lipids. b) SHS patterns of the same liposomes in pure $\mathrm{H}_{2} \mathrm{O}$. The scattering pattern originates from the overall transmembrane asymmetry in the orientational distribution of water molecules around the lipids (as illustrated in the cartoons). 


\section{Conclusions}

In summary nonlinear light scattering and imaging experiments offer a great multidimensional toolbox to investigate aqueous nanoscale systems and interfaces. Future studies aimed at characterizing water droplets, and their supercooling/freezing behavior are possible. More complex membrane and lipid droplet studies can be investigated as well as more fundamental energy transfer processes (by for example implementing a pump beam). With a sizeable amount of information available on the interfacial structure of nanoscale droplet systems it becomes possible to more accurately determine the physics and chemistry behind the difference in nanoscopic and macroscopic systems. The unprecedented sensitivity of second harmonic scattering to the orientational order of water is a great tool to investigate the active role water plays in stabilizing interfaces and solutions. It will be used in the future to further investigate biological systems as well as other fundamental surface processes. Especially the ability to determine surface potentials has great applications in biology, chemistry, and physics. In addition, many chemical reactions depend on it, for example the transfer of electrons across a surface. A combination with imaging also has promising applications, as many membrane processes depend on surface potentials (for example (action) potentials).

\section{Acknowledgements}

This work is supported by the Julia Jacobi Foundation, the Swiss National Science Foundation (grant numbers 200021_140472, 200021-146884, 200021_163210), the European Research Council (grant numbers 240556 and 616305), and the European Commission, Research Executive Agency Marie Curie Actions 'FINON' (ITN-2013-607842).

Received: March 30, 2017
[1] A. W. Adamson, A. P. Gast, 'Physical chemistry of surfaces' Wiley-interscience: Sidney, 1997.

[2] R. J. Hunter, 'Foundations of Colloid Science', Oxford University Press: Sidney, 2002.

[3] P. Ball, Chem. Rev. 2008, 108, 74.

[4] 'Membrane Hydration: The Role of Water in the Structure and Function of Biological Membranes', Ed. E. A. Disalvo, Springer, 2015.

[5] E. Bjornehohn, M. H. Hansen, A. Hodgson, L. M. Liu, D. T: Limmer, A. Michaelides, P. Pedevilla, J. Rossmeisl, H. Shen, G. Tocci, E. Tyrode, M. M. Walz, J. Werner, H. Bluhm, Chem. Rev. 2016, 116, 7698.

[6] L. G. M. Pettersson, R. H. Henchman, A Nilsson, Chem. Rev. 2016, 116, 7459.

[7] H. B. de Aguiar, J. S. Samson, S. Roke, Chem Phys. Lett. 2011, 512, 76.

[8] H. B. de Aguiar, R. Scheu, K. C. Jena, A. G. F. de Beer, S. Roke, Phys. Chem. Chem. Phys. 2012, 14, 6826.

[9] H. B. de Aguiar, A. G. F. de Beer, S. Roke, $J$. Phys. Chem. B 2013, 117, 8906.

[10] R. Scheu, S. Roke, J. Phys. Chem. B 2014, 118 , 3366.

[11] N. Smolentsev, Y. X. Chen, K. C. Jena, M. A Brown, S. Roke, J. Chem. Phys. 2014, 141, $18 \mathrm{C} 524$.

[12] S. Roke, O. Berg, J. Buitenhuis, A. van Blaaderen, M. Bonn, Proc. Nat. Acad. Sci. 2006, 103, 13310.

[13] N. Smolentsev, W. J. Smit, H. J. Bakker, S. Roke, submitted 2017.

[14] N. Smolentsev, C. Lutgebaucks, H. I. Okur, A. G. F. de Beer, S. Roke, J. Am. Chem. Soc. 2016, 138,4053 .

[15] N. Gomopoulos, C. Lutgebaucks, Q. C. Sun, C. Macias-Romero, S. Roke, Opt. Express 2013, 21,815 .

[16] A. G. F. de Beer, J. S. Samson, W. Hua, Z. Huang, X. Chen, H. C. Allen, S. Roke, J. Chem. Phys. 2011, 135, 224701.

[17] S. Roke, G. Gonella, Annu. Rev. Phys. Chem 2012, 63, 353 .

[18] A. G. F. de Beer, Y. Chen, R. Scheu, J. C. Conboy, S. Roke, S. J. Phys. Chem. C 2013 , 117, 26582.

[19] G. Gonella, C. Lutgebaucks, A. G. F. de Beer, S. Roke, J. Phys. Chem. C 2016, 120, 9165.

[20] A. G. F. de Beer, S. Roke, J. Chem. Phys. 2016 , $145,044705$.

[21] R. Vacha, S. Roke, J. Phys. Chem. B 2012, 116, 11936.

[22] G. Tocci, C. Liang, D. M. Wilkins, S. Roke, M. Ceriottit, J. Phys. Chem. Lett. 2016, 7, 4311.

[23] C. Lutgebaucks, G. Gonella, S. Roke, Phys. Rev. B 2016, 94, 195410.
[24] C. Macias-Romero, M. E. P. Didier, P. Jourdain, P. Marquet, P. Magistretti, O. B. Tarun, V. Zubkovs, A. Radenovic, S. Roke, Opt. Express 2014, 22, 31102

[25] C. Macias-Romero, M. E. P. Didier, V. Zubkovs, L. Delannoy, F. Dutto, A. Radenovic, S. Roke, Nano Lett. 2014, 14, 2552.

[26] C. Macias-Romero, V. Zubkovs, S. Wang, S. Roke, Biomed. Opt. Express 2016, 7, 1458.

[27] Y. Chen, H. I. Okur, N. Gomopoulos, C. MaciasRomero, P. S. Cremer, P. B. Petersen, G. Tocci, D. M. Wilkins, C. W. Liang, M. Ceriotti, S. Roke, Sci. Adv. 2016, 2, e1501891.

[28] C. Liang, G. Tocci, D. M. Wilkins, A. Grisafi, S Roke, M. Ceriotti, Phys. Rev. B 2017, submitted.

[29] D. M. Wilkins, D. E. Manolopoulos, S. Roke, M. Ceriotti, J.Chem. Phys. 2017, in press.

[30] H. I. Okur, Y. Chen, D. M. Wilkins S. Roke, Chem. Phys. Lett. (Frontiers) 2017, submitted.

[31] R. Vacha, S. W. Rick, P. Jungwirth, A. G. F. de Beer, H. B. de Aguiar, J. S. Samson, S. Roke, J. Am. Chem. Soc. 2011, 133, 10204.

[32] K. C. Jena, R. Scheu, S. Roke, Angew. Chem. Int. Ed. 2012, 51, 12938.

[33] J. S. Samson, R. Scheu, N. Smolentsev, S. W. Rick, S. Roke, Chem. Phys. Lett. 2014, 615, 124.

[34] Y. Chen, K. C. Jena, S. Roke, J. Phys. Chem. C 2015, 119,17725 .

[35] W. J. Smit, N. Smolentsev, J. Versluis, S. Roke, H. J. Bakker, J. Chem. Phys. 2016, 145, 044706.

[36] H. B. de Aguiar, M. L. Strader, A. G. F. de Beer, S. Roke, J. Phys. Chem. B 2011, 115, 2970.

[37] R. Scheu, Y. X. Chen, H. B. de Aguiar, B. M Rankin, D. Ben-Amotz, S. Roke, J. Am. Chem. Soc. 2014, 136, 2040.

[38] J. N. Israelachvili, 'Intermolecular and surface forces', Academic Press, 1991.

[39] R. Scheu, Y. Chen, H. B. de Aguiar, B. M. Rankin, D. Ben-Amotz, S. Roke, J. Am. Chem. Soc. 2014, 136, 2040.

[40] W. Kunz, Curr. Opin. Colloid Interface Sci. 2010, 15,34

[41] W. Kunz, Pure Appl. Chem. 2006, 78, 1611.

[42] P. Jungwirth, P. S. Cremer, Nature Chem. 2014, $6,261$.

[43] R. Scheu, Y. Chen, M. Subinya, S. Roke, J. Am. Chem. Soc. 2013, 135, 19330

[44] C. Lutgebaucks, C. Macias-Romero, S. Roke, $J$. Chem. Phys. 2017, 146, 044701.

[45] Y. Chen, K. C. Jena, C. Lutgebaucks, H. I. Okur, S. Roke, Nano Lett. 2015, 15, 5558.

[46] H. I. Okur, Y. Chen, N. Smolentsev, E. Zdrali, S. Roke, J. Phys. Chem. B 2017, 121, 2808. 